\title{
Análisis industrial de la reducción del óxido de hierro mediante la inyección de finos de carbón al horno eléctrico de arco $^{(\bullet)}$
}

\author{
A.N. Conejo ${ }^{(*)}$, R. Torres $^{(* *)}$ y E. Cuellar ${ }^{(* *)}$
}

\begin{abstract}
Resumen Se realizó un estudio a escala industrial en hornos eléctricos de arco (HEA) empleando una carga metálica compuesta al $100 \%$ de hierro esponja (DRI - Direct Reduction Iron), el cual evalúa el grado de oxidación del sistema metal-escoria. El consumo de energía disminuye con la inyección de oxígeno, sin embargo, el grado de oxidación de la escoria se incrementa. Para reducir el grado de oxidación de la escoria y mantener volúmenes altos de oxígeno inyectado es necesario: a) optimizar la práctica de inyección de finos de carbón, b) incrementar el porcentaje de carbono del DRI, c) hacer que trabaje el HEA por encima de una concentración crítica de carbono disuelto, tanto en el metal como en la escoria, y d) emplear temperaturas bajas, del orden de $1.650{ }^{\circ} \mathrm{C}$. Se propone un método rápido para definir el carbono del DRI en función de su grado de metalización y de la cantidad de oxígeno a inyectar. Para optimizar la práctica de inyección de finos de carbón es crítica la posición de la lanza de inyección, con objeto de asegurar un mayor tiempo de residencia de las partículas de carbón.
\end{abstract}

Palabras clave: Óxido de hierro. Finos de carbón. Formación de escoria espumosa.

\section{Industrial study of iron oxide reduction by injection of carbon particles into the electric arc furnace}

\begin{abstract}
An industrial study was conducted in electric arc furnaces (EAF) employing $100 \%$ direct reduced iron to evaluate the oxidation level of the slag-metal system. Energy consumption is decreased by injecting gaseous oxygen, however, slag oxidation also increases. In order to reduce the extent of oxidation while keeping a high volume of the oxygen injected, it is required: a) to optimize the carbon injection practice, b) to increase the carbon concentration of sponge iron, c) to operate with soluble carbon in both the metal and the slag beyond a critical level and d) to employ a low temperature profile, on average $1,650^{\circ} \mathrm{C}$. A method to define the proper amount of carbon in sponge iron which considers their metallization as well as the amount of oxygen injected is proposed. The position of the lance is critical in order to optimize the practice of carbon injection and assure a better residence time of the carbon particles within the furnace.
\end{abstract}

Keywords: Iron oxide. Carbon injection. Slag foaming.

\section{INTRODUCCIÓN}

Una de las innovaciones tecnológicas más importantes de la industria siderúrgica ha sido la separa-

\footnotetext{
$(\bullet \quad$ Trabajo recibido el día 5 de octubre de 1998 y aceptado en su forma final el 22 de enero de 1999.

(*) Instituto Tecnológico de Morelia. Av. Tecnológico 1500. 58120 Morelia, Mich. (México) Tel + (43) 138755 ext 208, 257

(**) Ispat Mexicana S.A. Francisco J. Mújica 1-B. 60950 Cd. Lázaro Cárdenas, Mich. (México).

(***) AIMCOR de México. Saturnino Herrán 77. Col. San José Insurgentes. 03900, México D.F. (México).
}

ción de las etapas de fusión y refino en dos reactores diferentes. En este esquema de producción de acero, el horno eléctrico de arco (HEA) se emplea únicamente como unidad de fusión, con lo que se ha logrado un incremento importante de productividad. Paralelamente, se ha logrado un incremento notable en la producción de acero por la vía del horno eléctrico, ruta que exige una fuerte disponibilidad de chatarra y cuyo déficit se estima (1) de 60 a $130 \times 10^{6}$ t para el año 2010 sobre la base de un crecimiento anual en la producción mundial de acero de 2,9\%. Para aliviar este déficit se contempla la construcción de más de 47 plantas 
productoras de materiales substitutivos de la chatarra, a partir de ahora y hasta el año 2000 , y cuya capacidad anual se estima en $105 \times 10^{6} \mathrm{t}$.

Ispat Mexicana (IMEXSA) trabaja sobre un esquema tecnológico que utiliza el horno eléctrico como unidad de fusión. Cuenta con dos plantas productoras de DRI (HYL-III y Megamod Midrex), lo que le permite reducir su dependencia de la chatarra.

El cambio estructural surgido con la especialización del horno eléctrico como unidad de fusión ha requerido modificaciones a la práctica metalúrgica tradicional. En el nuevo escenario, para incrementar la productividad del HEA se requiere lo siguiente:

- Incrementar la rapidez de fusión.

- Minimizar el consumo de energía, reduciendo las pérdidas térmicas y controlando el perfil de temperatura durante la fusión.

- Optimizar el balance de carbono y oxígeno suministrados al HEA asociado con un uso intenso de la inyección de oxígeno.

La inyección de oxígeno es una vía importante para aumentar la velocidad de fusión de la carga metálica, debido a que aporta energía química resultante de la oxidación del acero; esta oxidación promueve las condiciones necesarias para la eliminación de fósforo. El efecto negativo de la inyección de oxígeno es la oxidación del sistema metalescoria. Badische Stahl Werke (BST) señala que el $75 \%$ del oxígeno reacciona con carbón produciendo $\mathrm{CO}$, y el resto reacciona con el metal, de acuerdo con las siguientes reacciones:

$$
\begin{aligned}
& \mathrm{C}_{(\mathrm{s})}+0,5 \mathrm{O}_{2(\mathrm{~g})}=\mathrm{CO}_{(\mathrm{g})}+2,7 \mathrm{kWh} / \mathrm{Nm}^{3} \mathrm{O}_{2} \\
& \underline{\mathrm{Me}}+0,5 \mathrm{O}_{2(\mathrm{~g})}=(\mathrm{MeO})+7,1 \mathrm{kWh} / \mathrm{Nm}^{3} \mathrm{O}_{2}
\end{aligned}
$$

El aprovechamiento del calor generado por la inyección de oxígeno es mayor comparado con la energía eléctrica, la cual tiene que ser transmitida del arco al baño líquido.

El factor más importante que limita el volumen de oxígeno inyectado es la metalización del DRI y, por consecuencia, la velocidad de fusión también se limita. Un DRI de baja metalización aporta más oxígeno, en forma de óxido de hierro $(\mathrm{FeO})$, requiriendo mayor suministro de carbono para equilibrar el oxígeno total; de lo contrario resulta un alto grado de oxidación tanto del metal como de la escoria. Utilizando altos contenidos de DRI de baja metalización en la carga metálica, se producen niveles tan altos como $40 \% \mathrm{FeO}$. El FeO de la escoria tiene efectos negativos muy intensos en todas las operaciones metalúrgicas subsecuentes, el aumento de $\mathrm{FeO}$ es mayor si la colada es tradicional, con canal de colada, debido al paso de escoria. Algunos de sus efectos más críticos son los siguientes:

- Incrementa el consumo de refractario, en especial el de la línea de escoria.

- Aumenta el consumo de desoxidantes debido al paso de escoria oxidada.

- Aumenta el tiempo de proceso en horno cuchara debido al mayor acondicionamiento requerido y menor rapidez de desulfuración.

- Obstruye las boquillas por el aumento de desoxidantes y menor grado de limpieza del acero.

- Disminuye el rendimiento metálico.

Por el contrario, cuando el grado de oxidación durante la colada es bajo $(<25 \%)$, se tienen problemas para desfosforar el acero. Las coladas con fósforo fuera de especificación no se aceptan para continuar con el proceso posterior en el horno de cuchara debido a que las condiciones de trabajo en ese reactor son adversas para eliminarlo. Es importante que funcione la colada con valores relativamente "altos" de FeO para asegurar el control de la desfosforación, sin llegar a que éste sea excesivo. Se busca trabajar con valores de $\mathrm{FeO}$ en la escoria de 26 a $31 \%$ durante la fusión. Valores superiores al $35 \% \mathrm{FeO}$ se consideran excesivos.

$\mathrm{La}$ tabla I muestra valores promedio de $\mathrm{FeO}$ en el vaciado durante los últimos cinco años. El promedio del $33 \%$ que resulta representa un problema importante en el funcionamiento de los hornos eléctricos de arco. En contexto, es difícil plantear una reducción severa del óxido de hierro, ya que un gran porcentaje (del orden del $75 \%$ ) de acero producido en planta es de aceros de bajo contenido de carbono, con un máximo de $0,06 \% \mathrm{C}$, y estos aceros requieren valores de carbono de colada menores de $0,06 \%$, lo que hace que las tasas de descarburación impuestas al sistema, así como el $\mathrm{FeO}$ durante la fusión, sean altos. No obstante lo anterior, con esas condiciones es posible lograr niveles de oxidación del orden de 28-30\% FeO, lo cual implica una disminución de 3-5 \% FeO. Para lograr este objetivo se deben equilibrar apropiadamente los insumos de carbono y oxígeno.

TABLA I.- Promedios anuales del grado de oxidación

TABLE I.- Annual average in the content of $\mathrm{FeO}$

\begin{tabular}{|c|c|r|c|c|}
\hline Año & $\mathrm{FeO}(\%)$ & $\begin{array}{c}\mathrm{O}_{2} / \mathrm{t} \\
\left(\mathrm{m}^{3}\right)\end{array}$ & $\mathrm{Kg}$ coque/t & $\begin{array}{c}\mathrm{Kg} \text { finos } \\
\mathrm{C} / \mathrm{t}\end{array}$ \\
\hline 1992 & 33,9 & & & \\
1993 & 30,5 & 4,3 & 4,0 & \\
1994 & 32,6 & 7,1 & 8,0 & \\
1995 & 34,0 & 11,5 & 8,3 & \\
1996 & 33,8 & 13,4 & 10,3 & 2,4 \\
\hline
\end{tabular}


La planta IMEXSA cuenta desde el inicio de operaciones en 1988 con un sistema de inyección de finos de carbón. A principios de los 90 no se disponía aún de una comprensión clara del fenómeno de formación de espuma y, por otro lado, esta técnica no estaba difundida en el país, a pesar de que desde finales del decenio de los 80 ya algunas plantas en el mundo realizaban esta práctica. Durante el arranque del sistema de inyección de finos de carbón se experimentaron problemas de explosiones que se atribuyeron en algunos casos a una mala práctica de inyección. Esto podría ser posible debido a que la solubilidad del carbono aumenta con una disminución de la temperatura. $\mathrm{Al}$ existir una gran cantidad de carbono disuelto asociado con un aumento rápido de la temperatura, la reacción de descarburación se hace muy violenta promoviendo explosiones. En 1994 se realizaron estudios para disminuir el contenido de $\mathrm{FeO}$ de la escoria mediante adiciones de coque por el sistema de alimentación continua (ALCO). Se concluyó (2) que la adición de coque, en cantidades de 1.000$2.000 \mathrm{~kg} / \mathrm{colada}$ era fundamental para reducir el contenido de FeO. En este estudio, el aspecto central fue analizar la disminución del consumo de energía promovida por la formación de escoria espumosa debido a las adiciones de coque y no por efecto de la inyección de finos de carbón.

El objetivo del presente trabajo consiste en evaluar las variables de proceso que afectan el grado de oxidación del sistema metal-escoria y reducir la concentración de $\mathrm{FeO}$ mediante optimización de la práctica de inyección de finos de carbón al horno eléctrico de arco.

\section{ANÁLISIS TEÓRICO}

\subsection{Formación de espuma}

Para reducir el óxido de hierro de la escoria se requiere inyectar finos de carbón, las reacciones generan burbujas de $\mathrm{CO}$ las cuales provocan la formación de espuma. Una vez que se forman, las burbujas tienden a coalescer con objeto de reducir la superficie específica total y con esto reducir la energía libre de todo el sistema para, posteriormente, flotar en el líquido. Su fuerza flotante está dada por la ley de Arquímedes:

$$
F=\frac{\pi d^{3}}{6}\left(\rho_{1}-\rho_{2}\right) g
$$

donde $d$ es el diámetro de la burbuja, $\rho_{1}$ es la densidad del líquido, $\rho_{2}$ la densidad del gas y $g$ la aceleración de la gravedad. Las burbujas menores a 0,2 $\mathrm{mm}$ de diámetro se comportan, en agua pura, esencialmente como esferas rígidas sólidas y su veloci- dad de flotación, en consecuencia, puede determinarse mediante la ley de Stokes:

$$
v=\frac{2}{9} \frac{g r^{2}\left(\rho_{1}-\rho_{2}\right)}{\mu}
$$

donde, $v$ es la velocidad terminal, $r$ el radio de la burbuja y $\mu$ la viscosidad del líquido. Las burbujas más grandes cambian su forma, al principio a una forma esferoidal y posteriormente a formas elipsoidales. La tendencia natural cuando dos burbujas entran en contacto es hacer que la película líquida entre ellas se adelgace y rompa, a menos que exista cierta oposición a estos procesos debido a la presencia de substancias tensoactivas en las interfases o que el tiempo de contacto sea muy corto.

Una espuma, al contener una fase gaseosa, retarda la transferencia de calor y este comportamiento se aprovecha positivamente en la producción de acero en el HEA, en el cual el consumo de energía es crítico para mejorar su eficiencia térmica. El HEA basa su principal aporte de energía en la transferencia del calor del arco eléctrico hacia la carga; sin embargo, bajo condiciones normales, la punta del electrodo está descubierta y permite radiar un gran porcentaje del calor generado. Estimaciones (3) sobre el porcentaje de energía aprovechado indican que se pasa de un $36 \%$ cuando el arco está desprotegido a un $100 \%$ cuando el arco está cubierto por la espuma. Al proteger el arco y reducir la intensidad del calor radiado hacia las paredes, el HEA se hace más eficiente térmicamente, reduciéndose globalmente el consumo de energía, logrando ahorros de $10-30 \mathrm{kWh} / \mathrm{t}$. Al disminuir el calor radiado disminuirá también el desgaste del refractario, especialmente en los puntos calientes. Otra ventaja de la formación de espuma en el HEA es la disminución del consumo de electrodos. La mayor parte del consumo de electrodos se debe a oxidación a altas temperaturas. Al cubrir el arco con la escoria espumosa, la oxidación del grafito se reduce considerablemente favoreciendo un menor consumo.

Ito et al. (4) desarrollaron una ecuación para el índice de espumamiento $(\Sigma)$ utilizando el análisis dimensional, que define este índice en función de las propiedades de la escoria y establece que la estabilidad de la espuma aumenta con un aumento en la viscosidad superficial de la escoria $(\mu)$ y una disminución tanto de la densidad $(\rho)$ como de la tensión superficial $(\gamma)$.

$$
\sum=b g \frac{\mu}{\sqrt{\rho \gamma}}
$$

donde $b$ es una constante.

Más recientemente, Fruehan (5) definió otro índice de espumamiento en el cual incluye el tamaño de las burbujas $\left(d_{\mathrm{b}}\right)$. 


$$
\Sigma=1,83 \frac{\mu^{0,5}}{\rho_{\mathrm{s}} \gamma^{0,2} d_{\mathrm{b}}^{0,9}}
$$

Mills y Keene ${ }^{(6)}$ revisaron valores de densidades y tensiones superficiales de escorias. Para el sistema $(\mathrm{CaO}+\mathrm{MgO})-\mathrm{SiO}_{2}-\mathrm{FeO}$ definieron las siguientes expresiones:

$$
\begin{gathered}
\rho=2,46+0,018(\% \mathrm{FeO}) ; \mathrm{g} \cdot \mathrm{cm}^{-3} \\
\gamma=0,75-0,57\left[\frac{\% \mathrm{SiO}_{2}}{100}\right]-0,137\left(\frac{\% \mathrm{FeO}}{100}\right) ; \text { dinas } \cdot \mathrm{cm}^{-1}
\end{gathered}
$$

Ogawa et al. (7) y Pak et al. (8) han demostrado que el espumamiento depende directamente del tamaño de las burbujas de $\mathrm{CO}$ y que escorias con una baja concentración de $\mathrm{FeO}$ no espuman. Cuando el tamaño de burbuja es inferior a $2 \mathrm{~mm}$ observaron un espumamiento intenso, observaron, asimismo, que la escoria de alto horno no espuma debido a su bajo grado de oxidación y alto contenido de azufre. Para reducir el tamaño de las burbujas, la tensión interfacial metal-escoria debe ser reducida. Un aumento de $\mathrm{FeO}$ reduce este valor según lo indica la ec. [8]. Al reducir la tensión interfacial, el ángulo de contacto disminuye, para el arreglo particular metal-gas-escoria, y un menor ángulo de contacto genera burbujas más pequeñas favoreciendo así el espumamiento. El aumento de $\mathrm{FeO}$ disminuye fuertemente la viscosidad de la escoria. Este efecto es desestabilizador de la espuma. Es de esperar, entonces, un efecto dual de este óxido. Fruehan (5 y 9) sostiene que el $\mathrm{FeO}$ disminuye la viscosidad superficial y que no es tensoactivo, por lo que no contribuye al efecto Marangoni, causante de la estabilidad de la espuma; en consecuencia, observó una disminución global del índice de espumamiento al aumentar la concentración de $\mathrm{FeO}$. En general, un aumento del $\mathrm{FeO}$ tiene un efecto mayor sobre la viscosidad que sobre la tensión superficial por lo que al aumentar su concentración disminuye el espumamiento de la escoria.

En relación con la presencia de partículas suspendidas en la escoria, éstas, en general, tienden a proporcionar mayor estabilidad a la espuma. La fluorita disminuye el espumamiento porque aumenta la solubilidad del $\mathrm{CaO}$ y reduce la cantidad de partículas sólidas. En el caso de las partículas de carbón, Kozakevitch (10) indica que "estas partículas en la escoria reducen el espumamiento debido a un fenómeno de superficie que rompe las burbujas de la espuma". Este comentario es sorprendente, ya que en la práctica real de formación de espuma en el HEA lo que se introduce son precisamente partículas de carbón para espumar la escoria. Los finos de carbón reducen la actividad termodinámica del óxido de hierro con lo cual se promueve el espumamiento. El fenómeno citado se aplica en el uso de agentes antiespumantes a convertidores BOF para colapsar los derrames (generación violenta de espuma). Kozakevitch explica que las partículas de carbón dentro de las membranas de líquido adyacente a las burbujas promueve una segunda generación de $\mathrm{CO}$, incrementando su presión parcial y, en consecuencia, un ascenso más rápido del gas con lo que se desestabiliza la espuma. No existen estudios experimentales publicados hasta la fecha que hayan estudiado esta dualidad. Es necesario investigar las condiciones con las cuales el carbón actúa como espumante y como antiespumante. Es posible, en principio, asignar un papel como agente espumante al carbón cuando este presenta alta reactividad (bajo contenido de materia volátil) y una granulometría fina.

En un estudio reciente, Kapoor et al. (11) indican una limitación en los estudios anteriores sobre espumamiento que es el haber realizado experimentación utilizando muy bajas velocidades de generación de gas $\left(0,01-0,10 \mathrm{~m} \cdot \mathrm{s}^{-1}\right)$ con respecto a las condiciones obtenidas en procesos tipo smelting $\left(0,18-2 \mathrm{~m} \cdot \mathrm{s}^{-1}\right)$. Bajo estas últimas condiciones, las propiedades del líquido ya no son relevantes y la espuma final será controlada por la energía externa suministrada y las condiciones de flujo de gas. El tamaño de las burbujas es del orden de varios centímetros de diámetro haciendo que los efectos de tensión superficial sean despreciables en comparación con otras fuerzas presentes en el sistema.

\subsection{Reducción de FeO}

La cinética de reducción del óxido de hierro de la escoria está influida fuertemente por la rapidez y condiciones de generación de burbujas de CO. Capodilupo et al. (12) indican que las burbujas de $\mathrm{CO}$ generadas en el baño líquido son más pequeñas y están más uniformemente distribuidas que las que se generan en la escoria; en consecuencia, producen un mejor espumamiento a su paso por la escoria. Ameling et al. (3) estiman que del total de gas $\mathrm{CO}$ generado, un $20 \%$ proviene de reacciones en el metal, y un $80 \%$ de reacciones en la escoria, y que lo ideal sería una relación contraria, es decir, más $\mathrm{CO}$ generado en el metal ya que, además de las ventajas señaladas durante su ascenso el refino y disolución de fundentes se incrementa.

A nivel laboratorio se ha efectuado una gran cantidad de estudios sobre la cinética de reducción del óxido de hierro. Philbrook et al. (13) realizaron en 1956 y 1967 dos estudios pioneros sobre este tema. En un grupo de experimentos se cuantificó la 
variación de $\mathrm{FeO}$ con el tiempo mientras el $\mathrm{FeO}$ de la escoria es reducido tanto por el carbono del refractario como por el carbono disuelto en el metal. Otro grupo de experimentos consistió en medir el FeO reducido exclusivamente por el carbono del refractario. La reacción de reducción considerada fue la reducción directa del $\mathrm{FeO}$ por el carbono disuelto en el metal, de la manera siguiente:

$$
(\mathrm{FeO})_{(1)}+\underline{\mathrm{C}}=\mathrm{Fe}_{(1)}+\mathrm{CO}_{(\mathrm{g})}
$$

la velocidad de reducción (consumo de $\mathrm{FeO}$ ), está dada por la siguiente expresión:

$$
-r_{\mathrm{FeO}}=k \cdot A \cdot \mathrm{C}_{\mathrm{FeO}}^{\alpha} \cdot \mathrm{C}_{\mathrm{C}}^{\beta}
$$

$$
\text { donde: } \begin{aligned}
-r_{\mathrm{FeO}} & =\text { velocidad de reducción de } \\
& \mathrm{FeO}, \\
= & \text { constante de la velocidad de } \\
& \text { reacción, } \\
\mathrm{C}_{\mathrm{FeO}}^{\alpha} \cdot \mathrm{C}_{\mathrm{C}}^{\beta}= & \text { concentración de } \mathrm{FeO} \text { y de } \\
& \text { carbono respectivamente, en } \\
& \text { el metal líquido, } \\
\mathrm{A} & =\text { área metal-escoria. }
\end{aligned}
$$

El dispositivo experimental se realizó de manera que la concentración de carbono disuelto en el metal correspondiera a la de máxima solubilidad. Bajo la condición de exceso de carbono de uno de los reactantes, la expresión anterior se reduce a lo siguiente;

$$
-r_{\mathrm{FeO}}=k^{\prime} \mathrm{C}_{\mathrm{FeO}}^{\alpha}
$$

o bien,

$$
\log \left(-r_{\mathrm{FeO}}\right)=\log k^{\prime}+\alpha \log \mathrm{C}_{\mathrm{FeO}}
$$

donde: $k^{\prime}=k A C_{\mathrm{C}}^{\beta}$. De esta manera, realizando mediante gráficos las pendientes de la curva velocidad de consumo de $\mathrm{FeO}$ en función de la concentración de $\mathrm{FeO}$, se determina el orden de reacción $(\alpha)$. Los resultados obtenidos indicaron un promedio de 2,0 y, por tanto, la reacción de reducción no es de tipo elemental. Utilizando el valor de $\alpha=2$, la constante de reacción se determina realizando el gráfico de la relación $\left(1 / \mathrm{C}-1 / \mathrm{C}_{0}\right)$ en función del tiempo, tomando como base la siguiente expresión:

$$
-\int_{\mathrm{C} 0}^{\mathrm{C}} \mathrm{C}_{\mathrm{FeO}}^{-2} \mathrm{dC}_{\mathrm{FeO}}=k^{\prime} \int_{0}^{\mathrm{t}} \mathrm{d} t
$$

La constante de reacción obtenida no presentó fluctuaciones importantes dentro del rango de 0,020-0,030, lo cual confirmó que la reacción se aproxima bastante a ser de segundo orden. Por otra parte, es importante considerar que esta constante de reacción es la suma de dos tipos de reacciones; primero, reacción del $\mathrm{FeO}$ con el carbono disuelto en el metal y segundo, reacción del $\mathrm{FeO}$ con el carbono del refractario. Se calcula la constante de reacción bajo condiciones de ausencia de metal líquido y a partir de ese valor se calcula el valor para la constante de reacción del metal líquido con la escoria. Los resultados indicaron que la constante de reacción del metal líquido con la escoria $\left(60 \times 10^{-5}\right)$ es cinco veces mayor que la constante de reacción de la escoria con el carbono del refractario $\left(12 \times 10^{-5}\right)$ a pesar de que el área de esta última interfase es mayor. Este resultado, insesperado, indica que la nucleación de burbujas en la interfase metal-escoria es mayor que en la interfase líquido-refractario.

El mecanismo de reacción que proponen Philbrook et al., meramente especulativo, consiste en difusión de iones de la masa de la escoria a la interfase de reacción gas-líquido, en esta interfase se forma una película de $\mathrm{Fe}^{0}$ y el oxígeno reacciona con el gas $\mathrm{CO}$ para formar $\mathrm{CO}_{2}$. Las reacciones se indican a continuación:

$\begin{array}{lll}\mathrm{FeO} & =\mathrm{Fe}^{2+}+\mathrm{O}^{2-} \\ \mathrm{Fe}^{2+}+2 \mathrm{e}^{-} & =\mathrm{Fe}^{0} \\ \mathrm{O}^{2-} & =\underline{\mathrm{O}}+2 \mathrm{e}^{-} \\ \mathrm{C}_{(\mathrm{s})}+\underline{\mathrm{O}} & =\mathrm{CO}_{(\mathrm{g})} \\ \mathrm{CO}_{(\mathrm{g})}+\underline{\mathrm{O}} & =\mathrm{CO}_{2(\mathrm{~g})}\end{array}$

La burbuja crece hasta que la relación $\mathrm{CO}_{2} / \mathrm{CO}$ se equilibra con el potencial de oxígeno que determina la concentración de $\mathrm{FeO}$ presente en la escoria. De estas etapas, el mecanismo controlante se asocia con la difusión de iones de la masa de la escoria a la interfase de reacción.

Fay Fun (14) investigó la velocidad de reducción del óxido de hierro líquido a $1.650^{\circ} \mathrm{C}$, con carbono sólido, en el rango de 5-70 \% FeO, utilizando el método del electrodo rotatorio de grafito. Al emplear relaciones teóricas para predecir la rapidez de reducción tomando como base la difusión molecular y gasificación del carbono, encontró que los resultados experimentales no correspondían a ninguno de esos casos, más bien se ubicaban dentro de la banda definida por ambos mecanismos. Fundamentándose en esta información, el autor propone que la reducción del $\mathrm{FeO}$ se efectúa por transporte convectivo, esto es, difusión de especies químicas asistida con la presencia de burbujas de CO.

Davies et al. (15) determinó la energía de activación involucrada en la reducción del $\mathrm{FeO}$, obteniendo un valor de $67 \mathrm{kcal} / \mathrm{mol}$. Este valor es muy alto en relación con la energía de activación de procesos cuyo mecanismo controlante es la difusión de 
especies gaseosas y, por tanto, descartó el transporte de especies gaseosas como mecanismo controlante.

Sugata et al. (16) introdujo gas CO a flujos mayores que los producidos durante la reducción del $\mathrm{FeO}$. La reducción del $\mathrm{FeO}$ fue inferior a la obtenida con carbono sólido y por ello, la reducción indirecta mediante $\mathrm{CO}$ no es el mecanismo controlante.

Más recientemente, Sheikhshab et al. (17) evaluaron la velocidad de reducción del FeO. En el análisis teórico resumen dos grupos de investigaciones, las que definen que la reducción del $\mathrm{FeO}$ se efectúa de manera directa mediante el carbono sólido y la reducción indirecta en la cual la reducción del $\mathrm{FeO}$ se efectúa mediante $\mathrm{CO}$, según se muestra en las siguientes reacciones:

$$
\begin{array}{ll}
\mathrm{FeO}_{(\mathrm{l})}+\mathrm{C}_{(\mathrm{s})} & =\mathrm{Fe}_{(\mathrm{l})}+\mathrm{CO}_{(\mathrm{g})} \\
\mathrm{FeO}_{(\mathrm{l})}+\mathrm{CO}_{(\mathrm{g})} & =\mathrm{Fe}_{(\mathrm{l})}+\mathrm{CO}_{2(\mathrm{~g})}
\end{array}
$$

Los resultados de este autor concuerdan con el primer tipo. La reacción [14] es una reacción global, endotérmica, la cual se puede describir como la suma de tres reacciones electroquímicas: reacciones [13], [14] y siguiente:

$$
\mathrm{C}_{(\mathrm{s})}+\mathrm{O}^{2-}=\mathrm{CO}_{(\mathrm{g})}+2 \mathrm{e}^{-}
$$

La reacción [14] indica la formación de pequeñas gotas de metal reducido mientras que la reacción [19] muestra el consumo de partículas de carbón por reacción con los iones oxígeno formando una fase gaseosa. Sheikhshab observó que al aumentar la extracción de $\mathrm{CO}$ del horno, la velocidad de reducción aumentaba debido a que el contacto de la superficie del carbono sólido con la escoria líquida se incrementa $\mathrm{y}$, por tanto, adquiere mayor importancia la reducción directa mediante carbono sólido. El autor plantea incluso que la no mojabilidad del carbón por la escoria permite generar burbujas más grandes lo que deja huecos mayores para el movimiento de la escoria y su reacción con las partículas de carbón.

\section{DESARROLLO EXPERIMENTAL}

La acería IMEXSA, en la que se realizó este estudio, dispone de cuatro hornos eléctricos (NKK) de ultraalta potencia, con un alto grado de automatización, capacidad nominal de $220 \mathrm{t}$, transformadores de 125 MVA, electrodos de 711,2 mm de diámetro (28") y diámetro interno de coraza de $7.200 \mathrm{~mm}$. Cada horno cuenta con un sistema automatizado de regulación de electrodos, sistema de alimentación continua de DRI, cal y coque. El sistema de colada es convencional mediante canal de colada.
El sistema convencional de inyección de finos de carbón está compuesto por un silo de doble tanque, la parte superior recibe el material y en la inferior se presuriza. La carga se realiza por lotes. El silo tiene una capacidad de $2,16 \mathrm{~m}^{3}$. El rango de flujos de descarga varía de $40-120 \mathrm{kgC} \cdot \mathrm{min}^{-1}$. El fluido de arrastre es aire a una presión constante de 3,5 bares. La manguera de descarga es de un diámetro de $38,1 \mathrm{~mm}(1,5$ "). La inyección se realiza con lanza por la puerta de escoria. La vida de la lanza depende de la posición en que esta se coloque; si se coloca en el centro de la puerta que es por donde la escoria sale con mayor velocidad, el desgaste es intenso y no dura más de una colada, pero si se coloca de lado puede durar varias coladas. Los problemas de este sistema están asociados, primero, con la carga manual por lotes del silo de almacenamiento, ya que al terminarse el material se requiere una grúa, la cual no siempre está disponible, segundo, el tiempo de residencia de los finos de carbón es corto comparado con otras regiones donde la escoria permanece más tiempo dentro del horno y, tercero, a la poca duración de las lanzas.

En febrero de 1997 se modernizó el sistema de transporte y almacenamiento en plataforma de los silos de finos de carbón con objeto de mantener un suministro constante a los hornos eléctricos. El transporte de los finos de carbón a los hornos eléctricos se efectúa por medios neumáticos; el aire empleado para transportarlo es sometido a un proceso de secado previo ya que la humedad es altamente perjudicial. La humedad en los finos de carbón causa problemas de taponamiento de las mangueras de inyección a los hornos. El silo de almacenamiento en cada horno es similar en diseño al silo convencional, cuenta con dos tanques. La capacidad del tanque inferior es de $1.250 \mathrm{~kg}$. Al consumirse los finos de carbón y llegar a la marca de $150 \mathrm{~kg}$ se detiene el suministro por aproximadamente un minuto para llenar y presurizar el tanque inferior. El valor del flujo de aire de arrastre se estima aproximadamente en $450 \mathrm{~L} \cdot \mathrm{min}^{-1}$.

El sistema convencional de inyección de oxígeno consiste en dos sistemas de lanzas de alimentación que, en conjunto, pueden manipular un total de cuatro lanzas. Las lanzas se introducen por las partes laterales del horno, con un desplazamiento respecto al centro de la puerta de escoria de $37^{\circ}$ y con un ángulo de inclinación de $30^{\circ}$. La planta ha funcionado con los dos sistemas utilizando únicamente una sola lanza por sistema. El diámetro de las lanzas es de $31,75 \mathrm{~mm}(1,25$ "). La velocidad máxima (variable) de inmersión de las lanzas es de $6 \mathrm{~m} \cdot \mathrm{min}$. La longitud de cada lanza puede oscilar de 5,5 a 7 $\mathrm{m}$. Los flujos típicos de oxígeno son de $2.250 \mathrm{~m}^{3} \cdot \mathrm{h}$ $\left(37,5 \mathrm{~m}^{3} \cdot \mathrm{min}\right)$ con una lanza, y de $4.500 \mathrm{~m}^{3} \cdot \mathrm{h}(75$ $\mathrm{m}^{3} \cdot \mathrm{min}$ ) con dos lanzas, trabajando con una presión 
de $13 \mathrm{~kg} \cdot \mathrm{cm}^{-2}$. En el decenio de los 80 se empleaban lanzas de acero al carbono sin recubrimiento que producían una velocidad de consumo muy alta y la necesidad de cambiar varias lanzas durante cada colada. A principios del decenio de los $90 \mathrm{se}$ introdujo en el país un tipo de lanzas con un recubrimiento metálico que aumenta su vida de trabajo. $\mathrm{El}$ recubrimiento a base de una aleación $\mathrm{Fe}-\mathrm{Al}$ confiere alta resistencia al desgaste a altas temperaturas. Con el sistema convencional descrito previamente las lanzas de inyección deben de reemplazarse varias veces durante un turno de trabajo lo que, además de generar demoras importantes, hace que la reacción de descarburación se detenga. En febrero de 1996 se instaló un nuevo sistema de lanzas de inyección de oxígeno enfriadas por agua que les proporciona una vida prolongada y con la característica adicional de acoplar de manera simultánea la inyección de finos de carbón. El nuevo sistema se instaló en los hornos eléctricos 2 y 4. El diseño, de la empresa Fuchs, consiste en dos lanzas sujetas mediante una estructura colocada en la zona lateral izquierda de los hornos, estas lanzas tienen un desplazamiento lineal de entrada y salida.

Cuando la temperatura del agua de enfriamiento excede de $56^{\circ} \mathrm{C}$, la lanza sale automáticamente como sistema de seguridad. La temperatura del agua oscila generalmente alrededor de $45{ }^{\circ} \mathrm{C}$. La lanza superior es la que realiza la inyección de oxígeno y la inferior la inyección de finos de carbón. El diseño Fuchs de la lanza de oxígeno dispone de una cabeza de cobre con un ángulo de inclinación de $25^{\circ}$, pero su vida resultó reducida, requiriendo cambios frecuentes. Esta limitación hizo necesario adquirir un nuevo diseño, de la empresa Berry, cuya característica es que dispone de una sola lanza, tanto para la inyección de oxígeno como para la inyección de finos de carbón.

Un problema del tipo de funcionamiento con lanzas refrigeradas con agua es la formación de costras por salpicaduras de material, cuando la lanza no se introduce apropiadamente dentro de la escoria impidiendo la salida de la lanza del horno. Con objeto de minimizar la formación de costras, se debe trabajar con la lanza sumergida dentro de la escoria y sin que esta haga contacto con el acero. Fuchs recomienda una profundidad de inmersión equivalente a 10 diámetros de la boqui1la. Para aumentar la vida de las lanzas se debe mantener una presión mínima del oxígeno de 10 $\mathrm{kg} \cdot \mathrm{cm}^{-2}$, si la presión es baja, el chorro se dispersa antes de salir de la boquilla y erosiona el tubo Venturi de salida.

La ventaja principal del sistema automatizado de inyección de oxígeno empleando lanzas refrigeradas por agua, es el aumento en las tasas de descarburación durante la colada, esto debido a la continuidad en el suministro de oxígeno lo que además contribuye a acelerar la fusión de la carga metálica debido al aporte de energía química. Una estimación teórica de la energía suministrada por las reacciones de oxidación es del orden de 3-4 kWh/ $\mathrm{Nm}^{3} \mathrm{O}_{2(\mathrm{~g})}$, por ejemplo, si se introducen $3.000 \mathrm{~m}^{3} \mathrm{O}_{2}$ por colada, se podría esperar un ahorro aproximado de energía de $50 \mathrm{kWh} / \mathrm{t}$, además de los beneficios como resultado de una mayor agitación del baño metálico.

El tipo de lanzas refrigeradas con agua tiene la limitante de no adicionar de manera independiente finos de carbón. En ocasiones, el grado de oxidación es excesivo y solamente se requiere continuar con la inyección de finos de carbón, en estos casos el operador saca la lanza e inyecta los finos de carbón sobre la superficie a fin de evitar que el orificio de inyección de oxígeno se obstruya. Esta práctica es altamente ineficiente ya que el sistema depurador de gases succiona parte del material además de dar lugar a errores en la interpretación de datos; alto consumo de finos de carbón implicaría mayor carbono disuelto en la escoria y menor grado de oxidación de la misma, sin embargo en muchos casos esta no es la tendencia observada debido a anomalías de este tipo.

Como medio alternativo para la oxidación del acero se dispone de un dosificador con mineral de hierro. Se emplea en casos de que el flujo total de oxígeno no sea suficiente para alcanzar las tasas de descarburación deseadas.

Para este estudio se obtuvieron muestras de DRI y escorias durante la fusión. El análisis químico de las muestras se realizó mediante equipos de fluorescencia de rayos-X (MRS 4000) y calorimetría $\left(\mathrm{LECO}^{\circledR} \mathrm{Al}-350\right)$. El equipo de fluorescencia de rayos- $\mathrm{X}$ determina de manera directa la composición por elementos y esta concentración se transforma al valor final de los diferentes compuestos. El calorímetro LECO basa su principio de medición en el calor de combustión de las diferentes sustancias, con este equipo se determina carbono y azufre.

\section{RESULTADOS}

\subsection{Caracterización física y química de finos de carbón}

El análisis químico y físico de los finos de carbón utilizado en este estudio corresponde a un solo tipo de carbón, representativo de los finos de carbón empleados cuyo proveedor es la empresa Aimcor. El origen de los finos de carbón es coque de petróleo. Al iniciar el estudio se disponía de tres marcas comerciales de finos de carbón. La comparación de resultados se ha publicado anteriormente (18), en la cual se seleccionó el producto actual por poseer mayor contenido de carbono fijo. 


\subsubsection{Análisis químico}

La tabla II muestra el análisis químico de carbono fijo, materia volátil, cenizas, azufre y humedad. En la misma tabla se indican valores sugeridos como especificación. Para determinar la cantidad de carbono fijo se requiere conocer previamente los contenidos de humedad, cenizas y materia volátil.

La humedad es aceptable. Con estos porcentajes de humedad, $<2 \%$, y si aún se presentan problemas de obturamientos es posible que existan problemas de secado del aire del sistema de inyección. El contenido de cenizas de la muestra es alto (11\%), lo cual no es común en el coque de petróleo. La ceniza del carbón disminuye negativamente la basicidad de la escoria, además de que consume energía durante su calentamiento, por lo tanto debe limitarse su presencia al mínimo.

El único componente de los finos de carbón que contribuye a espumar la escoria es el carbono fijo. El material Aimcor fue el que presentó el mayor porcentaje de carbono fijo de los carbones disponibles.

\subsubsection{Granulometría}

Los resultados de granulometría se indican en la tabla III. Debido en parte a que los finos de carbón no están considerados como insumo crítico, no existe formalmente una especificación en planta. La empresa Voest Alpine (19) sugiere que la mayor parte de los finos de carbón $(\geq 90 \%)$ se ubique en el rango de 1-2 mm con un máximo de finos $(\leq 10$ $\%$ ) entre 0,05-1 mm. Este rango es similar al sugerido por un estudio japonés en el cual se obtuvo una mayor velocidad de reducción del FeO con partículas de $1 \mathrm{~mm}$ en comparación con partículas de 0,5 $\mathrm{mm}$; sin embargo, a diferencia de lo anterior, Ameling et al. (13) realizaron una comparación entre dos grupos de finos de carbón, 0-1 mm y 2-5 mm, y concluyeron que el material más fino es más recomendable debido a que la espuma se forma más rápidamente y de manera más intensa. El producto Aimcor contiene el $100 \%$ de finos con tamaño de partícula $<1 \mathrm{~mm}$.

TABLA II.- Composición química de finos de carbón (\%)

TABLE II.- Chemistry of carbon particles (\%)

\begin{tabular}{|c|c|c|c|c|c|}
\hline Material & $\begin{array}{c}\text { Materia } \\
\text { volátil }\end{array}$ & Cenizas & C fijo & Humedad & S \\
\hline $\begin{array}{c}\text { Especifi- } \\
\text { cación }\end{array}$ & $\leq 3$ & $\leq 3$ & $\geq 93$ & $\leq 1$ & $\leq 0,5$ \\
Aimcor & 3,06 & 11,37 & 85,57 & 2,1 & 1,6 \\
\hline
\end{tabular}

(*) Carbono fijo en base seca.
TABLA III.- Granulometría de finos de carbón

TABLE III.- Grain size of carbon particles

\begin{tabular}{|c|c|c|c|c|c|c|c|}
\hline Malla & 18 & 20 & 30 & 70 & 100 & 200 & -200 \\
mm & 1,00 & 0,84 & 0,59 & 0,21 & 0,149 & 0,074 & $-0,074$ \\
$\begin{array}{c}\text { Carbooster } \\
(\%)\end{array}$ & 8,0 & 1,2 & 3,6 & 6,8 & 13,2 & 38,0 & 29,2 \\
\hline
\end{tabular}

El tamaño de partícula es de gran importancia en el proceso de formación de espuma ya que para que ésta se lleve a cabo se requiere, entre otras cosas, lo siguiente:

- Cantidad mínima de FeO en la escoria ( 25\%).

- Inyección de carbono al acero o a la escoria.

- Temperatura alta (superior a $1.550^{\circ} \mathrm{C}$ ).

- Tiempo de residencia superior al crítico.

De estas condiciones, el tiempo mínimo de residencia debe ser tal que cada partícula se disuelva, reaccione y flote en la superficie. Si el tamaño es "grande" y no alcanza a disolverse y reaccionar al $100 \%$, entonces su eficiencia se verá disminuida y la formación de espuma será deficiente. Por el contrario, y debido a la formación de una esfera de gas producida por el aire de arrastre en contacto con la escoria, en las partículas "muy pequeñas" el momento mínimo para atravesar la esfera de gas y hacer contacto con la escoria no es suficiente, con lo cual su eficiencia disminuye. El tamaño ideal va a depender de una serie de factores específicos del proceso de inyección tales como la presión del aire de arrastre (la que le imparte el momento de penetración), el espesor de la capa de escoria, el flujo de sólidos inyectados, la profundidad de penetración de la lanza, etc.

\subsubsection{Superficie especifica}

La tabla IV muestra el resultado obtenido en la determinación de la superficie especifica mediante la técnica B.E.T. utilizando un equipo de adsorción. El resultado indica un área superficial pequeña en comparación con la superficie especifica de un catalizador $\left(>10 \mathrm{~m}^{2} \mathrm{~g}^{-1}\right)$.

TABLA IV.- Superficie específica de finos de carbón TABLE IV.-Specific surface area of carbon particles

\begin{tabular}{|c|c|}
\hline Material & $\mathrm{A}_{\mathrm{sp}}\left(\mathrm{m}^{2} / \mathrm{g}\right)$ \\
Aimcor & 4,31 \\
\hline
\end{tabular}




\subsubsection{Otras propiedades}

a): La morfología de las partículas de carbón se analizó mediante microscopia electrónica de barrido (SEM). Las partículas exhiben una geometría esférica combinada con partículas de forma poliédrico-irregular. Es ventajoso contar con partículas esféricas ya que se presenta así un frente de reacción más uniforme,

b) La densidad y la conductividad térmica no fueron evaluadas experimentalmente; sin embargo, estos valores son muy característicos y se encuentran en tablas. La tabla V muestra densidades típicas de diversos materiales, observándose que la densidad del grafito es menor que la de la escoria y, por tanto, tiene una alta tendencia a flotar. La densidad teórica del grafito policristalino es de $2,26 \mathrm{~g} / \mathrm{cm}^{3}$ en ausencia de poros. Debido a la existencia de poros, la densidad disminuye. El tamaño de los poros en algunos grafitos varía entre 0,2-1,0 $\mu \mathrm{m}$,

c) La conductividad térmica del carbón es alta según se desprende de la tabla V. Esto es importante ya que un aumento de finos de carbón en la escoria contribuirá a aumentar su conductividad térmica y mejorar el precalentamiento del DRI que penetra la escoria.

\subsection{Grado de oxidación del sistema metal- escoria}

Una de las características más importantes de IMEXSA es el hecho de trabajar con una carga metálica compuesta prácticamente por un $100 \%$ de DRI. Esta operación la realizan muy pocas plantas en el mundo. Trabajar de esta forma presenta diversos problemas, los cuales se complican cuando la metalización del DRI es baja. El DRI contiene com-

TABla V.- Densidad y conductividad térmica de varios materiales

TABLE V.-Density and thermal conductivity of several materials

\begin{tabular}{|c|c|c|}
\hline Material & $\begin{array}{c}\text { Densidad, } \\
\mathrm{g} / \mathrm{cm}^{3}\end{array}$ & $\begin{array}{c}\text { Conductividad } \\
\text { térmica, } \\
\left(\mathrm{cal} / \mathrm{m}^{\circ} \mathrm{C} \mathrm{s}\right)\end{array}$ \\
\hline Goma natural & $0,81-0,91$ & 0,033 \\
Aluminio & $2,57-2,92$ & $27-55$ \\
Acero & $7,47-7,83$ & $3-8,5$ \\
Escorias & $2,50-3,50$ & - \\
$\begin{array}{c}\text { Grafito } \\
\text { (policristalino) }\end{array}$ & $1,50-2,00$ & $27-39$ \\
\hline
\end{tabular}

ponentes mineralógicos secundarios, tales como $\mathrm{SiO}_{2}, \mathrm{CaO}, \mathrm{MgO}$, etc., que presentan un alto punto de fusión y requieren de energía extra para fundirse. La metalización promedio del DRI durante 1997 fue de $89 \%$. En fechas posteriores a la realización de este estudio, la metalización promedio del DRI aumentó de 89 a $94 \%$, lo que ocasiona una mejora en la vida del refractario, al pasar de un promedio de vida de 350 coladas, en la línea de escoria, a 490 coladas. Este resultado muestra claramente la fuerte influencia que ejerce la metalización en la eficiencia de la acería.

Es importante señalar que, aunque el parámetro de control industrial del grado de oxidación de la escoria es el FeO de fusión, es igual de importante controlar el $\mathrm{FeO}$ durante la fusión. El FeO durante la etapa de fusión es el que determina el rendimiento metálico, a diferencia del FeO de fusión, el cual sólo representa las condiciones de fin de colada, además de que puede ser mayor debido al aumento de temperatura de colada y al incremento de oxígeno suministrado. Un contenido alto de $\mathrm{FeO}$ a fin de colada no necesariamente es indicativo de un contenido alto de $\mathrm{FeO}$ durante la etapa de fusión. Los resultados analizados corresponden a condiciones de fusión y de fin de colada, estos fueron correlacionados con las variables de proceso empleadas con objeto de definir las condiciones que permitan disminuir el grado de oxidación de la escoria a valores en el orden del $30 \%$. La figura 1 muestra el efecto de la metalización sobre la concentración del FeO. Al aumentar la metalización, el FeO disminuye. El porcentaje promedio del FeO del DRI fue de 8-12\%. La información de la figura sugiere que para lograr valores inferiores al $30 \% \mathrm{FeO}$ en la escoria, la metalización del DRI debe ser de al menos $92 \%$. Al aumentar la metalización se logra

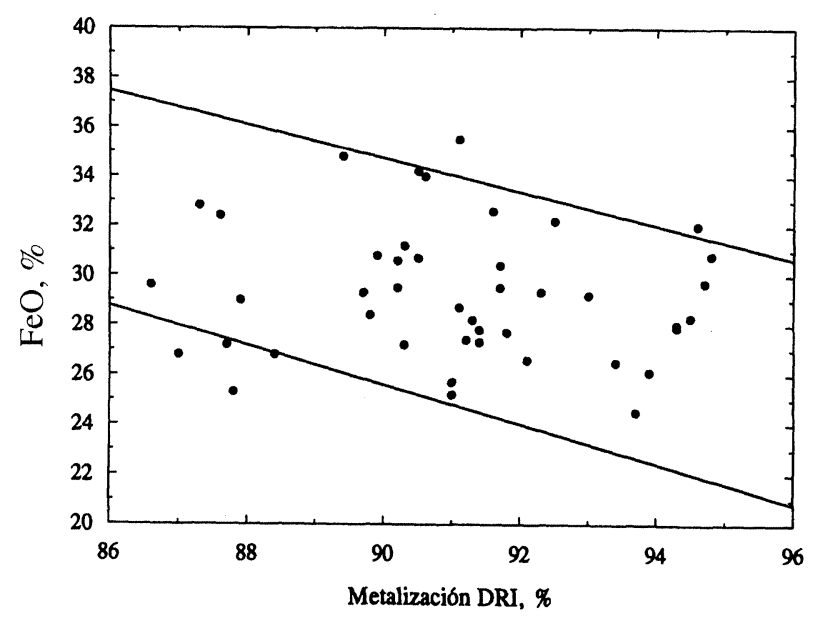

FIG. 1.- Efecto de la metalización del DRI sobre la concentración de $\mathrm{FeO}$ antes de la colada.

FIG. 1.- Effect of DRI-metallization on $\mathrm{FeO}$ before casting. 
también una disminución de la energía requerida para fundir. Cuando la metalización fue inferior al $90 \%$, se obtuvieron consumos de energía de 700$750 \mathrm{kWh} / \mathrm{t}$, y al aumentar la metalización ese valor se redujo a $650 \mathrm{kWh} / \mathrm{t}$.

Además de la metalización del DRI, la temperatura de operación y el flujo de oxígeno inyectado también afectan al grado de oxidación de la escoria.

El rango de temperaturas de vaciado fluctuó de 1.620 a $1.700{ }^{\circ} \mathrm{C}$. Al aumentar la temperatura aumenta el oxígeno disuelto en el acero y simultáneamente aumenta el FeO de la escoria (Fig. 2). Se observa que un $60 \%$ de las coladas se cuelan con temperaturas superiores a $1.650^{\circ} \mathrm{C}$, lo cual repercute en un exceso de la concentración de oxígeno. A fin de reducir el nivel de oxidación, la temperatura de colada debe ser inferior a $1.650^{\circ} \mathrm{C}$. El problema por el cual se trabaja con altas temperaturas de colada se atribuye a dos factores; primero, al tipo de colada convencional el cual exige compensar las pérdidas de calor durante el vaciado, equivalente a $50{ }^{\circ} \mathrm{C}$, y segundo, a la acumulación de cucharas en la nave de colada, que implica largos tiempos de espera con lo cual la temperatura del acero disminuye, agravándose aún más por la exposición de la superficie de la cuchara a la atmósfera. Es recomendable trabajar en esta etapa final con arco corto $(\approx 15 \mathrm{~cm})$ ya que si se trabaja con arco largo $(\approx 30 \mathrm{~cm})$ la poca altura de la escoria provoca que quede el arco descubierto radiando energía a las paredes, con lo cual se reduce la eficiencia térmica del horno.

La primera medición del grado de oxidación de la escoria que posee el operario de horno es la concentración de oxígeno en partes por millón (ppm). Una correlación de datos entre $\underline{\mathrm{O}}-\mathrm{FeO}$ indica que si la concentración de oxígeno disuelto en el acero

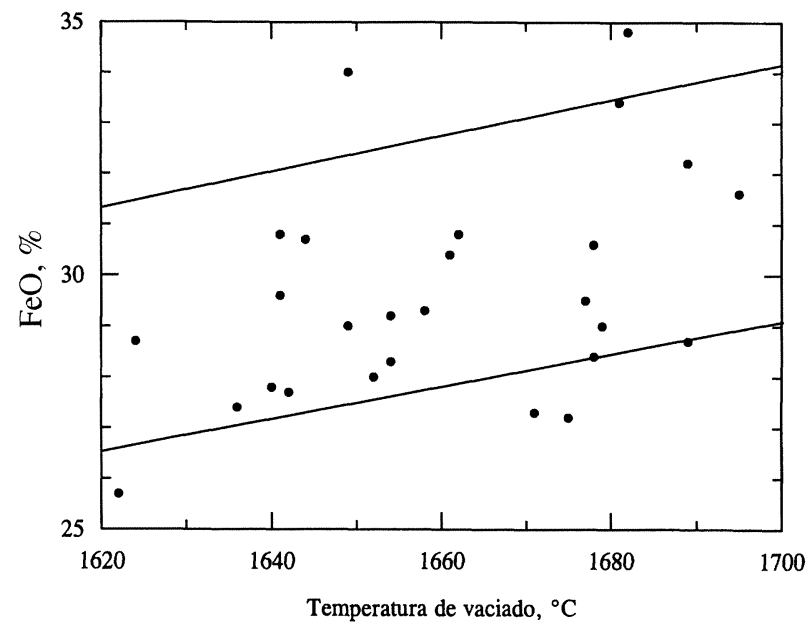

FIG. 2.- Efecto de la temperatura de colada sobre el grado de oxidación de la escoria.

FIG. 2.-Effect of casting temperature on FeO. excede 1.000 ppm, entonces, el FeO de la escoria es superior al $30 \%$.

La figura 3 muestra cómo afecta la inyección de oxígeno en el grado de oxidación de la escoria. El consumo promedio de oxígeno en planta durante los últimos años ha sido del orden de $10 \mathrm{~m}^{3} / \mathrm{t}$, que no es un valor alto si se considera que existen plantas en el mundo que trabajan con consumos del orden de $35 \mathrm{~m}^{3 / \mathrm{t}}$. Al exceder un consumo de 10 $\mathrm{m}^{3} / \mathrm{t}$, el FeO de la escoria rebasa el $30 \%$. La tendencia indica que para valores de oxígeno de 20 $\mathrm{m}^{3} / \mathrm{t}$, el ahorro de energía obtenido varió de 1 a 5 $\mathrm{kWh} / \mathrm{m}^{3} \mathrm{O}_{2}$. Al aumentar el volumen de oxígeno inyectado, la oxidación del sistema metal-escoria se hace más crítica de controlar. Para equilibrar el flujo de oxígeno adicional inyectado es necesario introducir carbono. De la forma y cantidad en que se adicione este carbono dependerá el grado final de oxidación. El equilibrio C-O determina la concentración de oxígeno durante la fusión en HEA. La figura 4 muestra que al colar el acero con una concentración igual o superior a $0,06 \% \mathrm{C}$, el valor correspondiente de $\mathrm{FeO}$ en la escoria es menor del $30 \%$. La dispersión de datos está influida por la temperatura. La restricción funcional para establecer una práctica metalúrgica con este nivel de carbono está relacionada con el nivel máximo permisible de carbono en las especificaciones. Existen aceros cuyo nivel máximo de carbono después de colado se sitúa en $0,05 \%$ y debido al contenido de carbono de las adiciones durante la colada, se debe colar con niveles del orden de 0,04\% C. En estos casos particulares, la variable más crítica de control del grado de oxidación es la temperatura, debiendo reforzar las precauciones sobre las condiciones de

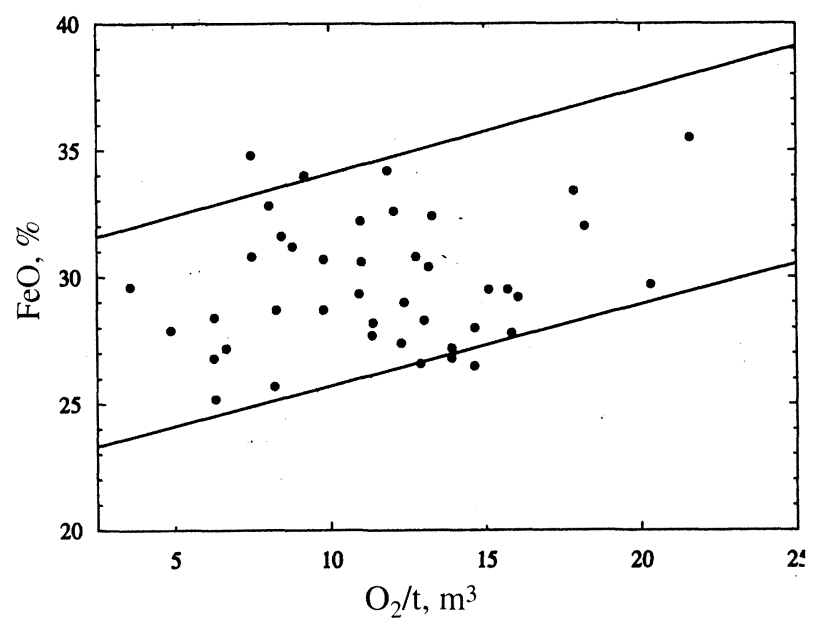

FIG. 3.- Relación entre el oxígeno inyectado al HEA y la concentración de FeO en la escoria antes de la colada.

FIG. 3.-Relationship between oxygen injected into the EAF and $\mathrm{FeO}$ in the slag before casting. 


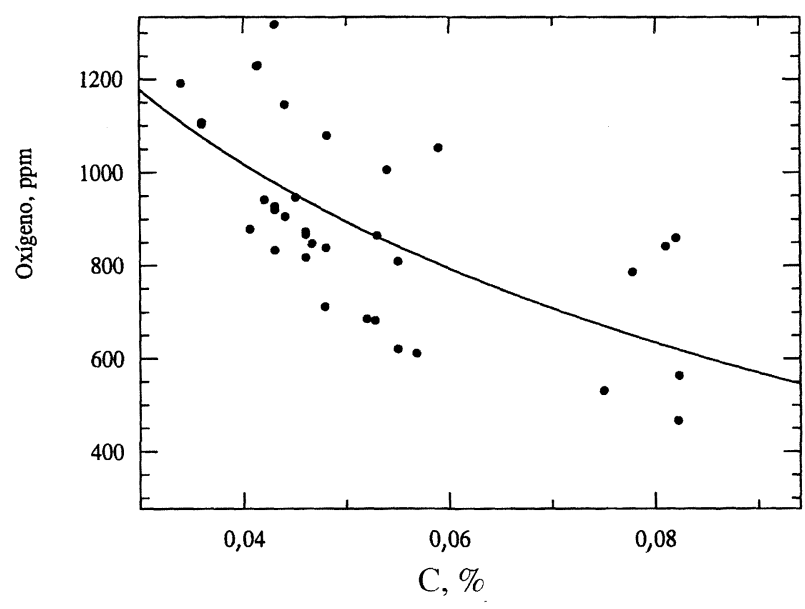

FIG. 4.- Relación carbono/oxígeno antes de la colada en el HEA.

FIG. 4.- Relationship C/O before casting from EAF.

colada. Los dos flujos principales de suministro de carbono al acero lo constituyen las adiciones de coque y el carbono contenido en el hierro esponja. El coque se adiciona por el sistema de alimentación continua y se realiza, principalmente, al inicio de la colada, momento en el que la escoria se encuentra generalmente muy oxidada y con alta temperatura, lo que hace que flujos elevados de coque generen una formación de espuma excesiva. Cuando el avance de la colada es superior al $30 \%$, la adición de coque actúa principalmente como agente espumante y su eficiencia es muy baja ya que cae sobre la superficie de la escoria. En planta, se ha evaluado la adición de diferentes cantidades de coque y se ha establecido que un valor total de $2.000 \mathrm{~kg}$ por colada $(9 \mathrm{~kg}$ de coque/t) es el más adecuado para controlar el grado de oxidación y reducir el consumo de energía.

Con relación a la forma de aportar carbono al sistema metal-escoria, es evidente que la mejor manera de aportar carbono al acero es mediante el carbono contenido en el DRI y no mediante adiciones de coque. La decisión de cómo aportar carbono involucra aspectos económicos, ya que existe un costo adicional del DRI para incrementar su contenido de carbono en los reactores de reducción directa durante la etapa final del proceso de reducción. El gas carburante en esta etapa final está formado por gas fresco, generalmente gas natural y otra parte por gas reciclado que contiene agentes carburantes. El uso adicional de gas natural para carburar incrementa el costo del DRI. Aunque no existe ningún estudio en planta que haya analizado el punto de equilibrio de este parámetro, se anticipan mayores ventajas a la incorporación del carbono en el DRI que en forma de coque, no sólo por tener una mayor eficiencia sino, también, porque el coque presenta la desventaja adicional de aportación de azufre, además de que si el contenido de carbono en el DRI es inferior al $2 \%$, el carbono resultante en el acero no es suficiente para controlar adecuadamente el grado de oxidación. Esta conclusión es válida para los valores de oxígeno inyectados y el grado de metalización del DRI empleado en este estudio. El contenido de carbono empleado en el DRI varió de 1,6-2,2 \%, más especialmente en el rango de $1,8-2,0 \% \mathrm{C}$.

Con relación a la forma en que se introduce el carbono para equilibrar el oxígeno, Midrex (20) propone limitar el contenido de carbono del DRI en virtud de que "un aumento del contenido de carbono disminuye las unidades de hierro" y "aumenta considerablemente el consumo de gas natural" disminuyendo la productividad de las plantas de reducción directa. Citan estudios efectuados en dos empresas en las cuales se varió el contenido de carbono en los rangos del 1,6-2,75 \% y del 1,0-3,5\%, con la conclusión de que por encima del $1,8 \% \mathrm{C}$ "no es económico debido a que los costos adicionales asociados con el incremento de carbono en el DRI no se justifican técnicamente ni se obtuvieron ahorros económicos en la acería". Los cálculos en que se basa Midrex no contemplan la inyección de oxígeno durante la fusión de la carga metálica y con esto, sus argumentos carecen de validez en el contexto de IMEXSA. Para las condiciones en planta es necesario estudiar cuál es el nivel óptimo de carbono en el DRI considerando el consumo específico de oxígeno durante la fusión

\subsection{Análisis de la inyección de finos de carbón}

La inyección de oxígeno y finos de carbón puede proceder de manera simultánea, sin embargo, para iniciar esta operación debe existir una cantidad mínima de escoria líquida para evitar que se produzca una fuerte radiación que afecta la vida de la(s) lanza(s) además de producir pérdidas de energía. En los casos en que se carga chatarra, los finos de carbón se inyectan a partir del momento en que termina de fundir la chatarra. La cantidad de gas oxígeno a adicionar se determina a partir del $\mathrm{FeO}$ inicial a $50 \mathrm{t}$.

Los resultados experimentales sobre el efecto de la inyección de finos de carbón en el consumo de energía se muestran en la figura 5 . El consumo de finos varió de 3 a $15 \mathrm{~kg} / \mathrm{t}(0,66-3,3 \mathrm{t} /$ colada), el cual es un rango muy amplio, esto se atribuye a las fluctuaciones en la calidad de la materia prima, principalmente metalización y contenido de carbono del DRI, los cuales variaron del 86-94 \% y 1,5-2,2 \%, respectivamente, así como a la cantidad de gas oxígeno inyectado, que varió de $5-20 \mathrm{~m}^{3} / \mathrm{t}$. El consumo promedio de energía para un consumo de finos de 


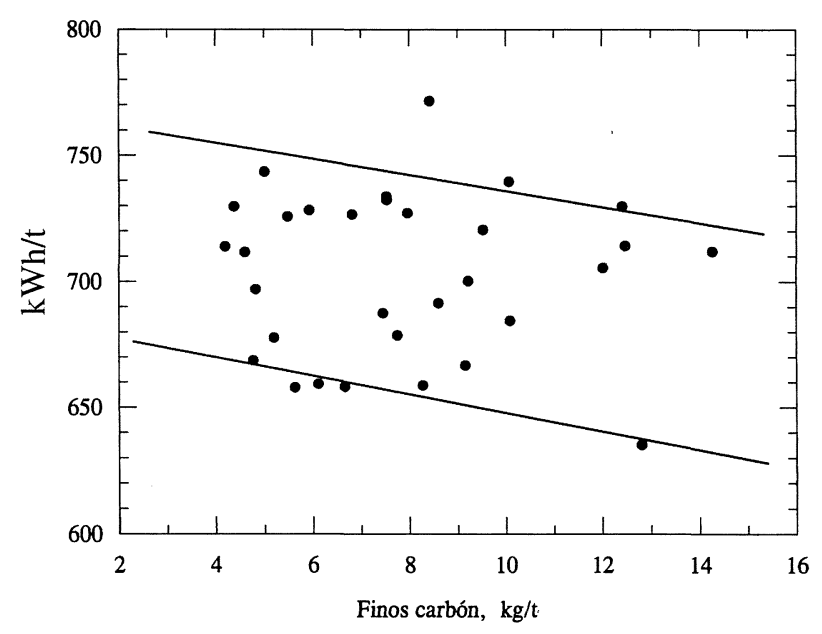

FIG. 5.- Efecto de la inyección de finos de carbón sobre el consumo de energía.

FIG. 5.- Effect of carbon inyection on energy consumption.

carbón de $5 \mathrm{kgC} / \mathrm{t}$ fue de $700 \mathrm{kWh} / \mathrm{t}$. Al aumentar el consumo de finos de carbón a $10 \mathrm{kgC} / \mathrm{t}$, el ahorro de energía disminuyó aproximadamente $20 \mathrm{kWh} / \mathrm{t}$. Este ahorro representa el efecto global de la reacción $\mathrm{C}-\mathrm{O}$, es decir, incluye también la disminución de energía por efecto de la inyección de oxígeno.

El consumo específico de finos de carbón debe ajustarse principalmente en función de la cantidad de oxígeno inyectado y del grado de metalización del DRI. El efecto que tiene la inyección de finos de carbón sobre el FeO final de la escoria se muestra en la figura 6. Se observa que al aumentar la cantidad de carbón inyectado disminuye la concentración de $\mathrm{FeO}$ en la escoria. El límite en la canti-

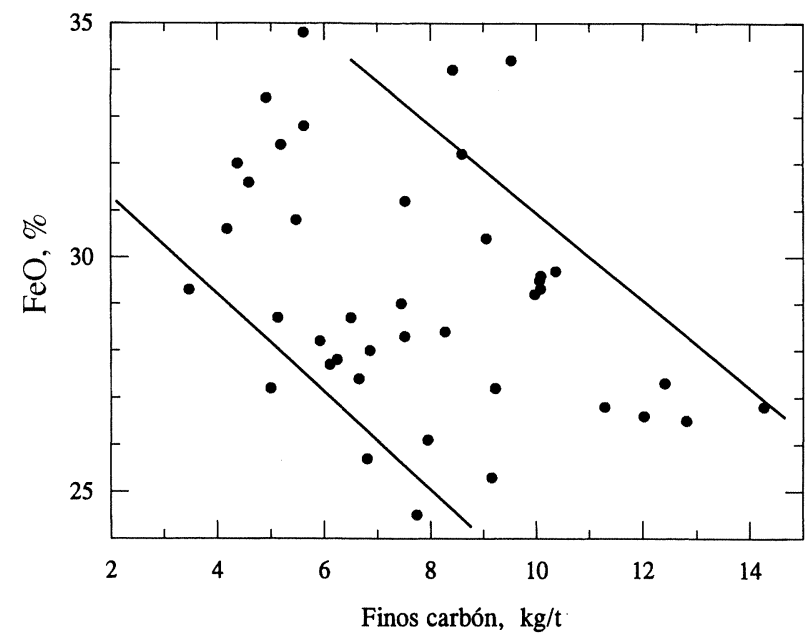

FIG. 6.- Efecto de la inyección de finos de carbón sobre el $\mathrm{FeO}$ de la colada.

FIG. 6.- Effect of carbon inyection on $\mathrm{FeO}$ before casting. dad de finos de carbón a inyectar debe ser tal que no carbure el acero por encima de $0,08 \% \mathrm{C}$, ya que una concentración superior disminuye considerablemente el grado de oxidación de la escoria afectando la desfosforación. La gráfica indica que se requiere un promedio de $7 \mathrm{~kg}$ finos $\mathrm{C} / \mathrm{t}$ (1,5 t/colada) para lograr menos del $30 \% \mathrm{FeO}$ en la escoria de colada. Se midió adicionalmente la concentración de carbono y $\mathrm{FeO}$ disueltos en la escoria a mitad de cada colada para un total de tres muestras, denominadas A, B y C, separadas por un tiempo de $10 \mathrm{~min}$. Los resultados de las tres muestras fueron similares, encontrando una correlación entre el carbono y el $\mathrm{FeO}$ disueltos en la escoria similar a la relación entre el carbono disuelto en el acero y el $\mathrm{FeO}$ de la escoria. En la figura 7 se muestra el efecto de la inyección de finos de carbón sobre la aportación de carbono a la escoria, para la muestra A. Utilizando el promedio de los datos experimentales anteriores, la inyección de $7 \mathrm{~kg}$ de finos de carbón/t de acero (equivalente a 1,5 t/colada) aporta aproximadamente $0,10 \% \mathrm{C}$ en la escoria. Estos promediós coinciden con la correlación obtenida en la figura 8 , que muestra la relación entre el carbono y $\mathrm{FeO}$ disueltos en la escoria. No obstante la dispersión de datos, se estima que para controlar el grado de oxidación de la escoria por debajo de $30 \% \mathrm{FeO}$, la concentración de carbono disuelto en la escoria debe ser alrededor del $0,10 \%$. La variabilidad en los datos experimentales se atribuye a tres factores: a) fluctuaciones en la práctica de inyección de finos de carbón que impiden una completa homogeneización del oxígeno y carbono, b) naturaleza heterogénea de la escoria y c) limitaciones de tipo cinético que impiden reaccionar por completo cada una de las partículas de carbón. En los hornos eléctricos 1

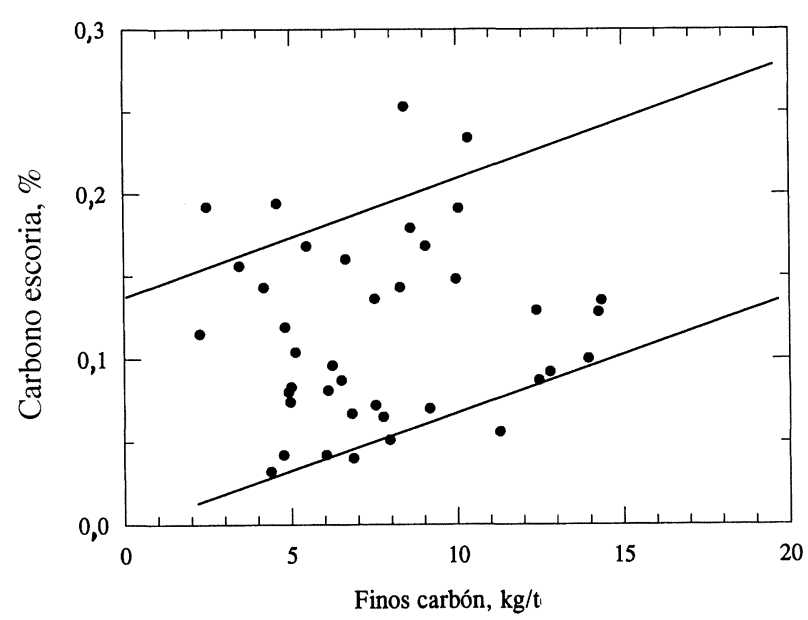

Fig. 7.- Efecto de la inyección de finos de carbón sobre la concentración de carbono en la escoria.

FIG. 7.- Effect of carbon inyection on carbon contribution in the slag. 


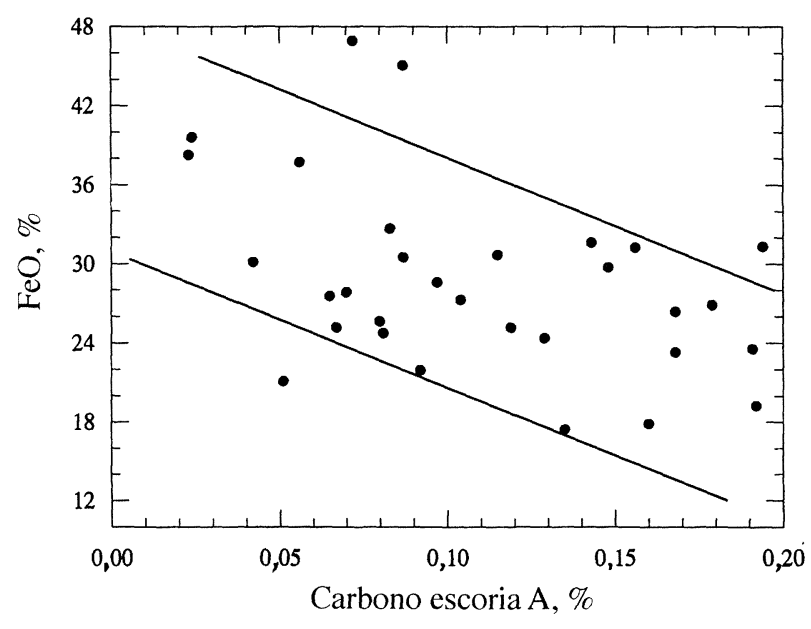

FIG. 8.- Relación $\mathrm{FeO} / \mathrm{C}$ en la escoria A.

FIG. 8.-Relationship FeO/C in the slag A.

y 3, la inyección de finos de carbón se realiza por la puerta de escoria; en esa zona la fuerza de arrastre es muy alta la cual empuja hacia al exterior las partículas de carbón, reduciendo su eficiencia. En los hornos 2 y 4 , la eficiencia se considera mayor debido a que la lanza de inyección se ubica en una región que permite mayor tiempo de residencia de las partículas de carbón y, además, el impulso adicional del chorro de oxígeno proporciona un mayor mezclado de este material.

Durante las pruebas, el flujo de finos de carbón osciló de $60-150 \mathrm{~kg} \mathrm{~min}^{-1}$. En principio, se estimaba funcionar con flujos del orden de $30 \mathrm{~kg} \mathrm{~min}^{-1}$, de acuerdo a estimaciones teóricas, pero por problemas operativos esto no fue posible. Un flujo de sólidos muy bajo ocasiona taponamientos de lanza; por otro lado, un flujo muy alto genera una formación de espuma descontrolada y la generación de llamas que afectan a la vida de las deltas.

Es deseable en el funcionamiento del HEA que la inyección de finos de carbón se realice durante más del $90 \%$ de la colada a fin de tener espuma que ayude en la reducción del consumo de energía eléctrica, lograr un ambiente menos contaminado debido al ruido y reducir el grado de oxidación de la escoria. El sistema de inyección instalado actualmente inyecta el material de manera continua. Otro sistema más atractivo y que permite mayor flexibilidad trabaja tomando como base los ciclos, es decir, de manera intermitente. Un ciclo consiste en una etapa de adición simultánea de finos de carbón y aire de arrastre; en la segunda etapa solamente se adiciona aire de arrastre. La duración de cada una de estas etapas depende de condiciones locales de cada empresa (21).

El uso de cantidades elevadas en la inyección de finos de carbón genera altos volúmenes de gas $\mathrm{CO}$ que tiende a postcombustionarse en la zona de las deltas cuando no existe equipo de postcombustión y las erosiona fuertemente. La erosión se agrava por la formación de llamas. Las llamas, indicativo de alto carbono, se pueden producir cuando la lanza de inyección de finos de carbón no está adecuadamente sumergida causando que los finos sobre la superficie de la escoria reaccionen para formar $\mathrm{CO}_{2}$.

\subsection{Equilibrio carbono-oxígeno suministrado al HEA}

En condiciones isotérmicas, una elevada concentración de óxido de hierro en la escoria es el resultado de una falta de equlibrio en los insumos de carbono y oxígeno al horno eléctrico de arco. Los resultados indican que el carbono real contenido en el DRI es superior al carbono estequiométricamente requerido, esto es, el DRI contiene la cantidad de carbono necesario para reducir su oxígeno presente en forma de $\mathrm{FeO}$. El carbono estequiométricamente requerido se calculó, con la siguiente fórmula (22): $\% \mathrm{C}_{\mathrm{DRI}}$ $=0,188(1-\mathrm{M}) \% \mathrm{Fe}_{\mathrm{t}}$, donde $\mathrm{M}$ es el grado de metalización. En un trabajo anterior (23) se definieron los cálculos para determinar el equilibrio $\mathrm{C}-\mathrm{O}$, empleando dos parámetros, el déficit de carbono y la relación $\mathrm{O} / \mathrm{C}$. El déficit de carbono resulta de un equilibrio de masa incluyendo reacciones químicas. El equilibrio $\mathrm{O} / \mathrm{C}$ se relaciona con la cantidad global de carbono y oxígeno introducidos al HEA. Por estequiometría, el equilibrio $\mathrm{O} / \mathrm{C}$ se alcanza para un valor de 1,33. Los resultados de ambos equilibrios se muestran en las figuras 9 y 10 . Como se indica en la figura 9, para asegurar que el grado de oxidación de la escoria se ubique en un máximo de $30 \%$

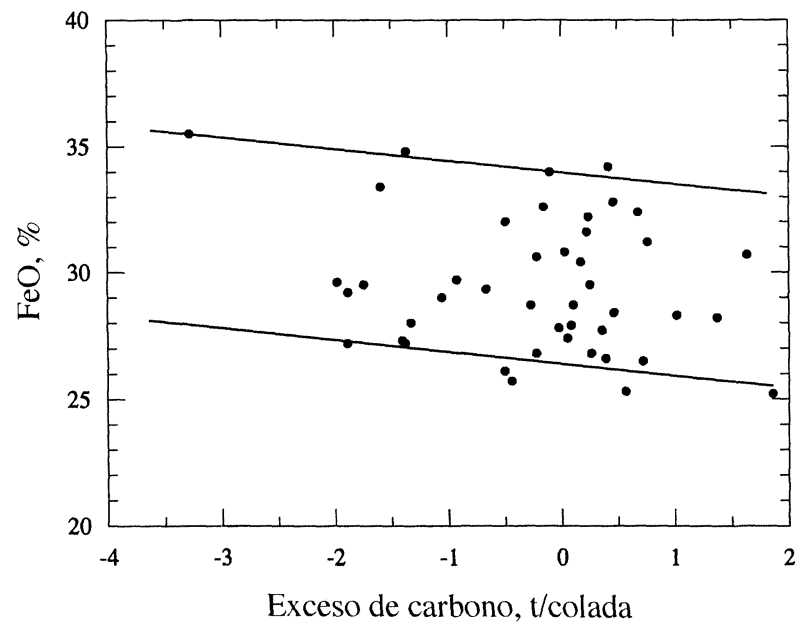

FIG. 9.- Efecto del "exceso de carbon" sobre el $\mathrm{FeO}$ de la colada.

FIG. 9.- Effect of "carbon excess" on $\mathrm{FeO}$ before casting. 


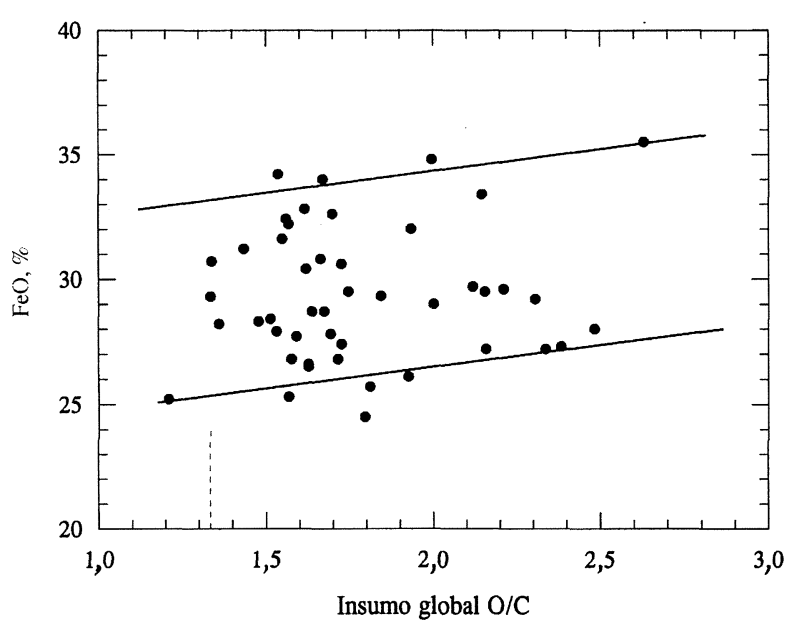

FIG. 10.- Efecto de la relación $\mathrm{O} / \mathrm{C}$ suministrada al HEA sobre la concentración final de $\mathrm{FeO}$ de la colada.

\section{FIG. 10.- Effect of the ratio O/C supplied into the EAF on $\mathrm{FeO}$ before casting.}

$\mathrm{FeO}$ es necesario trabajar en condiciones de exceso de carbono en la carga metálica, en cantidades del orden de $1 \mathrm{t} \mathrm{C/colada} \mathrm{(4,5} \mathrm{kg} \mathrm{C/t).} \mathrm{La} \mathrm{necesidad} \mathrm{de}$ trabajar con un exceso de carbono en la carga metálica es consecuencia del desequilibrio termodinámico bajo condiciones de planta. Si este carbono adicional se introduce a través del DRI, se requiere entonces que el porcentaje de carbono actual se incremente. La cantidad promedio de DRI empleado en este estudio fue de 1,9·251 t DRI/colada, resultando 4,8 t C/colada aportadas por el DRI. Si se suma la tonelada adicional requerida, el carbono deseado en el DRI debe ser de 2,3\% con objeto de lograr el exceso de carbono necesario que permita reducir la concentración de $\mathrm{FeO}$ por debajo de $30 \%$. Esta es una conclusión global válida para el grado de metalización del DRI empleado; a mayor metalización menor será el requerimiento, tanto de oxígeno como del carbono en exceso.

Con respecto a la relación $\mathrm{O} / \mathrm{C}$ se observa en la figura 10 que todos los datos experimentales se ubican en condiciones globales de exceso de oxígeno. Como se indicó anteriormente, para un valor teórico de 1,33 de la relación $\mathrm{O} / \mathrm{C}$ se obtiene el balance global carbono-oxígeno. Para este valor, el promedio de $\mathrm{FeO}$ de fin de fusión es de $28 \%$. Se observa que limitando la relación O/C a un máximo de 1,7, el $\mathrm{FeO}$ no rebasa el $30 \%$.

La tabla VI muestra la aportación de oxígeno, proveniente del oxígeno inyectado y de oxígeno del DRI, por colada. En el estudio actual, el volumen promedio de oxígeno inyectado fue de $10 \mathrm{~m}^{3} \mathrm{O}_{2} / t$, equivalente a $3,1 \mathrm{t} /$ colada. Comparando este valor con el oxígeno aportado por el DRI se observa que este oxígeno puede rebasar en un $100 \%$ al aportado
TABLA VI.- Oxígeno aportado por el DRI y gas- $\mathrm{O}_{2}$ inyectado (colada $220 \mathrm{t}$ )

TABLE VI.- Oxygen coming from DRI and blowed (220 ton heat size)

\begin{tabular}{|c|c|c|c|c|}
\hline \multicolumn{2}{|c|}{ Oxígeno inyectado } & \multicolumn{3}{|c|}{$\begin{array}{c}\text { Oxígeno aportado } \\
\text { por el DRI }\end{array}$} \\
\hline $\begin{array}{c}\mathrm{O}_{2} / \mathrm{t} \\
\left(\mathrm{m}^{3}\right)\end{array}$ & $\mathrm{O}(\mathrm{t})$ & Met\% & $\begin{array}{c}\mathrm{O}_{\mathrm{DRI}} \\
(\%)\end{array}$ & $\mathrm{O}(\mathrm{t})$ \\
\hline 10 & 3,1 & 86 & 3,5 & 6,8 \\
12 & 3,8 & 90 & 2,5 & 6,3 \\
15 & 4,7 & 94 & 1,5 & 3,7 \\
\hline
\end{tabular}

por el gas-oxígeno cuando la metalización del DRI es baja (86-90\%). Es posible, tomando como base estos datos proponer un método rápido para equilibrar el carbono y el oxígeno. Consideremos, por ejemplo, el caso de que se introduzcan $10 \mathrm{~m}^{3} \mathrm{O}_{2} / \mathrm{t} \mathrm{y}$ $251 \mathrm{t}$ de DRI en la carga metálica con una metalización del $86 \%$, de acuerdo con la tabla, para una colada de $220 \mathrm{t}$, se aportan 9,6 t de oxígeno. Utilizando el valor de 1,7 para el equilibrio C-O y si se introduce todo el carbono a través del DRI, entonces el porcentaje de carbono en el DRI debe ser de $2,25 \% \mathrm{C}$, de acuerdo con los siguientes cálculos:

$\mathrm{t}$ de oxígeno al HEA $=\mathrm{t}$ de oxígeno inyectado + $t$ de oxígeno en DRI $=9,6$

$\mathrm{t}$ de carbono para equilibrar oxígeno $=\mathrm{t}$ de oxígeno $/ 1,7=5,64$

$\% \mathrm{C}$ en DRI $=(\mathrm{t}$ de carbono DRI $/ \mathrm{t} \mathrm{DRI}) \times 100=$ $(5,64 / 251) \times 100=2,25 \% \mathrm{C}$

\section{CONCLUSIONES}

- La productividad del horno eléctrico de arco depende de la velocidad de fusión de la carga metálica, ésta es acelerada mediante la inyección de oxígeno, sin embargo, el grado de oxidación del sistema metal-escoria se incrementa afectando negativamente el proceso mismo de fusión y operaciones posteriores de refino secundario. La inyección de finos de carbón dentro de la escoria permite que, de manera simultánea a la oxidación del acero, se produzca la reducción del óxido de hierro. Un nivel alto de oxidación del sistema metal-escoria (FeO-ppm O) es el resultado de: primero, un balance inadecuado de los insumos de carbono y oxígeno; segundo, limitaciones de diseño que impiden una completa acción de los finos de carbón sobre todo el volumen de la escoria, y tercero, altas temperaturas de operación, superiores a $1.650{ }^{\circ} \mathrm{C}$. Por otra parte, la recuperación del hierro metálico 
reducido se ve limitada por consideraciones de tipo cinético que impiden el descenso de las partículas al baño metálico antes de que salga la escoria del horno.

- El factor más importante de control del $\mathrm{FeO}$ de la escoria es el equilibrio carbono-oxígeno (CO). El rango obtenido del $\mathrm{FeO}$ cuando la carga metálica está equilibrada varía del 27-34\%. Para lograr niveles del $\mathrm{FeO}$ inferiores al $30 \%$ es necesario trabajar con una carga metálica conteniendo un exceso de carbono, que debe introducirse, en principio, como parte del DRI. Para el tipo de carga empleado en las coladas estudiadas y los niveles de oxígeno inyectados, se requiere de $1 \mathrm{t} \mathrm{C/colada,} \mathrm{equivalente} \mathrm{a} \mathrm{cargar} \mathrm{DRI} \mathrm{conte-}$ niendo $2,3 \% \mathrm{C}$.

- La variable de proceso que tiene mayor influencia sobre el $\mathrm{FeO}$ de la escoria es la concentración de carbono en el baño metálico. Al emplear valores superiores a $0,06 \% \underline{\mathrm{C}}$ se asegura una escoria de fusión con bajo nivel de $\mathrm{FeO}$ y, asimismo, una baja concentración de ppm $\underline{\mathrm{O}}$ en el baño metálico. Para mantener niveles superiores a $0,06 \% \mathrm{C}$ durante la colada (sin llegar a reducir el $\mathrm{FeO}$ por debajo de $25 \%$ ) se concluyó que, además del valor indicado de carbono en el DRI $(2,3 \%)$, se deben realizar adiciones de coque de $10 \mathrm{~kg} / \mathrm{t}$ al inicio de la colada y finos de carbón durante la colada de $7 \mathrm{~kg} / \mathrm{t}$, sin llegar a exceder la cantidad de finos para evitar un deterioro acelerado de las deltas. Utilizando el valor indicado de finos de carbón se obtiene en promedio de $0,10 \% \mathrm{C}$ disuelto en la escoria. El carbono de la escoria es otra variable de control del FeO.

- Mediante un cálculo global se ha determinado que si la metalización del DRI es menor del 90 $\%$, la cantidad de oxígeno introducida por este material puede rebasar hasta más del $100 \%$ la cantidad de oxígeno introducido mediante la inyección de gas-oxígeno. Conociendo la metalización y concentración de carbono del DRI se puede determinar la máxima cantidad de gasoxígeno a introducir, utilizando el factor $\mathrm{O} / \mathrm{C}$ de 1,7 encontrado como válido para las condiciones de planta. Por otra parte, si lo que se desea es inyectar un volumen máximo de oxígeno, el cálculo permite definir el carbono del DRI para evitar una sobreoxidación del sistema metalescoria. Dependiendo de la cantidad y eficiencia del coque es posible inyectar una cantidad adicional de oxígeno. En todo momento, el carbono del baño líquido es el parámetro de control a seguir.

- La disminución del FeO de fin de fusión en planta, implementando los controles descritos en este trabajo, fue del $33 \%$ como media de años anteriores al $31 \% \mathrm{FeO}$ en 1997.

\section{REFERENCIAS}

(1) Hogan, P. Steel Technol. Int., 1997: 2.

(2) Lule, R. Memorias del Encuentro Nacional de Siderurgicos, San Luis Postosí-SLP, México, 1994.

(3) Ameling, D., Petry, J., Sittard, M., Ulrich, W. y Wolf, J. Stahl Eisen, 106 (11), 1986: 45-50. BISI Translation 25147.

(4) Ito, K. y Fruehan, R.J. Iron Steelmaker, 16 (8), 1989: 55-60.

(5) Fruehan, R.J., Ito, K. y Ozturk, B. Steel Res., 60 (3+4), 1989: 129-137.

(6) Mills, K.C. y KeEne, B.J. Int. Met. Rev., 32, 1987: 1.

(7) Yugi Ogawa y NaOKi Tokumitsu. Observation of slag foaming by X-Ray fluoroscopy", Proc. $6^{\text {th }}$ Int. Iron and Steel Congress, ISIJ, Nagoya (Japón), 1990: 47-152.

(8) PaK, J.J., Min, D.J. y You, B.D. Slag foaming phenomena and its supression techniques in BOF steelmaking process. Steelmaking Conference Proceedings. EE.UU., 763-769.

(9) Fruehan, R.J. Met. Mat. Trans., 28B, oct. 1997: 751.

(10) Kozakevitch, P.P. Formation and destruction of foams and emulsions in iron and steelmaking, Proc. The Physical Chemistry of Steelmaking. Ed. J.F.Elliot, EE.UU., jun. 1956: 89-92.

(11) KAPOOR, A. y Irons, G.A. ISIJ Int. 37 (9), 1997: 829-838.

(12) Capodilupo, D., Masucci, P., Brascugli, G y Angelis, V. de. Operating improvements in electric steel production on the Terni EAF after introduction of slag foaming practice", Proc. 6th Int. Iron and Steel Congress, ISIJ, Nagoya (Japón), 1990: 98-103.

(13) Philbrook, W.O. y Kirkbride, L.D. J. Metals, mar. 1956: 351

(14) FAY Fun, Met. Trans. B, sep 1970, 2537-2541.

(15) Davies, M.W., Hazeldean, G.S.F. y SMith, P.M. Kinetics of reaction between iron oxide slags and carbon, Richardson Conference, 1973, IMM, 95-107.

(16) Sugata, M., Sugiyama, T. y Kondo, S. Trans. AIME, dic. 1964: 1609-1614.

(17) Sheikhshab, M., Fukuda, M., Ito, Y., Yamada, S. y SANO, M. ISIJ Int., 33 (11), 1993: 1125-1130.

(18) Conejo, A. y Magaña, F. Estabilidad de escoria espumosa del HEA. XIX Simposio Nacional de Siderurgia, ITM, Morelia, Mich. (México), 1997: 2.1-2.6.

(19) Mittag, P. Voest Alpine, 1997, Comunicación personal

(20) MIDREX Suppliers Seminar, Toronto Canadá, Marzo 21, 1998

(21) VAZQUEZ, S. Inyección periódica automatizada de grafito durante la fusión y afino en la fabricación de acero en HEA. Memorias del XIV Encuentro Nacional de Siderúrgicos, Ixtapa, Gro (México), 2-3 oct. 1997.

(22) Conejo, A.N. y Viramontes, R. IX Curso nacional de siderurgia; Tecnología de reducción directa. ITM, oct. 1996, Morelia Mich. (México).

(23) CONEJO, A.N. y LoPEZ, F. Análisis del grado de oxidación de la escoria, balanceando los insumos de carbón y oxígeno al horno eléctrico de arco. Memorias del XIV Encuentro Nacional de Siderurgicos, Ixtapa, Gro (México), 2-3 oct. 1997: 1-12. 